\title{
Resolved Observations of Transition Disks
}

\author{
Simon Casassus ${ }^{1,2}$ \\ ${ }^{1}$ Departamento de Astronomía, Universidad de Chile, Casilla 36-D, Santiago, Chile \\ 2Email: scasassus@u.uchile.cl
}

(Received November 09, 2015; ACCEPTED February 04, 2016)

\begin{abstract}
Resolved observations are bringing new constraints on the origin of radial gaps in protoplanetary disks. The kinematics, sampled in detail in one case-study, are indicative of non-Keplerian flows, corresponding to warped structures and accretion which may both play a role in the development of cavities. Disk asymmetries seen in the radio continuum are being interpreted in the context of dust segregation via aerodynamic trapping. We summarise recent observational progress, and describe prospects for improvements in the near term.
\end{abstract}

Keywords: protoplanetary discs - submillimetre: stars - planet-disc interactions - planets and satellites: formation (stars:) circumstellar matter - stars: formation

\section{INTRODUCTION}

Circumstellar disks evolution is an aspect of stellar formation (Shu, Adams, \& Lizano 1987; Zuckerman 2001; Williams \& Cieza 2011; Dunham et al. 2015). In Class II young-stellar-objects, the dissipation of the protostellar envelope exposes a pre-main-sequence star surrounded by a gaseous accretion disk. In this framework, the spectralenergy-distributions (SEDs) provide constraints on radial disk structure. Central cavities (and gaps) are inferred in so-called transition disks (and pre-transitional disks, Espaillat, Calvet, D’Alessio, Hernández, Qi, Hartmann, Furl 2007, TDs hereafter), whose structure is being refined with resolved observations.

This review provides a selection of structures seen in TDs, with an emphasis on the radio domain. Insights on the origin of the cavities based on TD demographics are summarised in the accompanying review by Owen (2015). We caution that resolved observations of TDs are affected by many biases, as they target the brightest sources with the largest cavities, so that they cannot be extrapolated to reflect the whole population (which is for now studied by complete photometric surveys, e.g. Cieza et al. 2012, 2015). Instead, such resolved observations inform on the range of possible astrophysical phenomena at work in disks.

The observational identification of abrupt disk inclination changes (Section 2) provides the information on disk orientation required to interpret the dynamics of residual gas inside TD cavities (Section 3). The outer rings surrounding the central cavities of most TDs resolved so far in the submillimetre continuum show non-axial symmetry, with intensity contrasts from a few to $\sim 100$ (Section 4). Complex spiral patterns are seen in the outer disks, which are also beginning to be resolved in molecular lines (Section 5). The exciting observation of giant protoplanet candidates opens an enormous research potential, since their formation is expected to carve a radial gap, but their detection in TDs is still far from systematic (Section 6). The inferred warped accretion flows, the dramatic non-axial symmetry of some outer disks, and the observed spiral patterns, motivate questions on their origins, their connection to embedded protoplanets, and on their role in disk evolution (Section 7).

\section{WARPS}

Warps, or variable inclination and orientation as a function of stellocentric distance, have long been suspected to occur in circumstellar disks. In this Section, we compile evidence for warps in young circumstellar disks in a broader context, with special attention to TDs and the case of HD 142527.

\subsection{Debris disks}

Warps are seen in debris disks, or Class III young stellar objects that have dissipated the left-over primordial gas. This is the case of the edge-on warp seen in $\beta$ Pic (Golimowski et al. 2006; Millar-Blanchaer et al. 2015). Another example is AU Mic (Wang et al. 2015; Boccaletti et al. 2015), and 
perhaps also HD 110058 (Kasper et al. 2015). The identification of warps in debris disks requires an edge-on view, and the lack of gaseous counterparts hampers reaching definitive conclusions on their structure, and so distinguish continuous warps from superimposed disks.

\subsection{Indications of warps in gas-rich disks}

Inner warps, or a tilted inclination at the disk centre, could lead to the photometric variations seen in some highinclination (so close to edge-on) Class II classical T-Tauri stars $^{1}$, such as in RY Lup (Manset et al. 2009), TWA 30 (Looper et al. 2010), and AA Tau (Bouvier et al. 2013). The light curve variability of AA Tau is well accounted for by a magnetically induced warp on scales of a few stellar radii or $\lesssim 0.1$ AU (Esau, Harries, \& Bouvier 2014), and part of the occulting structure could extend beyond 1 AU (Schneider et al. 2015). In the NGC 2264 open cluster, up to $~ 40 \%$ of young and gas-rich cTTs with thick inner disks present AA Tau-like variability (Alencar et al. 2010), suggesting that such inner warps represent a fairly common phase in the early evolution of circumstellar disks (McGinnis et al. 2015). Light curve variability on weeks and months timescales, due to obscuring material, has also been reported in the TDs T Cha (Schisano et al. 2009) and GM Aur (Ingleby et al. 2015).

In TDs, warps have also been hinted at in the form of significant inclination changes in observations with different angular resolutions. For example in GM Aur, with a stellar mass $\lesssim 1 \mathrm{M}_{\odot}$, Hughes et al. (2009) propose a central warp to explain the small change in disk position angle ${ }^{2}$ of $11^{\circ} \pm 2^{\circ}$ when comparing the major axis of the submillimetre continuum sampled at 0.3 arcsec, with that of the $\mathrm{CO}(3-2)$ kinematics $^{3}$ sampled at 2 arcsec. Likewise, Tang et al. (2012) explain that in AB Aur $\left(\sim 2 \mathrm{M}_{\odot}\right)$, the inclination inferred from near-IR interferometry $\left(i \sim 20^{\circ}\right.$ within the central AU; Eisner et al. 2004) is close to that sampled over $20 \mathrm{AU}$ scales, and lower than sampled in coarser beams $\left(i \sim 36^{\circ}\right.$ outside 70 AU; Piétu, Guilloteau, \& Dutrey 2005). Hashimoto et al. (2011) also conclude, from polarised-differential-imaging (PDI) in the near-IR, that the inner regions of AB Aur are warped, given the varying inclinations in a double ring structure, dropping from $\sim 43^{\circ}$ to $\sim 27^{\circ}$ over 90 to $200 \mathrm{AU}$ in radius. Tang et al. (2012) relate the warped structure inferred from their molecular line data with a residual infalling envelope.

Yet, another example of such inclination changes with angular scale is seen in MWC $758\left(\sim 2 \mathrm{M}_{\odot}\right)$, where near-IR interferometry (with VLTI+AMBER, Isella et al. 2008 ) yields an inclination of $30^{\circ}-40^{\circ}$ in the central $\mathrm{AU}$, while Submillimeter Array (SMA) observations in $\mathrm{CO}(3-2)$, with a 0.7 arcsec beam spanning over $100 \mathrm{AU}$, yield

\footnotetext{
${ }^{1}$ I.e. $\lesssim 1 \mathrm{M}_{\odot}$ stars undergoing accretion.

${ }^{2}$ The intersection of the plane of the disk with that of the sky.

${ }^{3}$ Assuming Keplerian rotation.
}

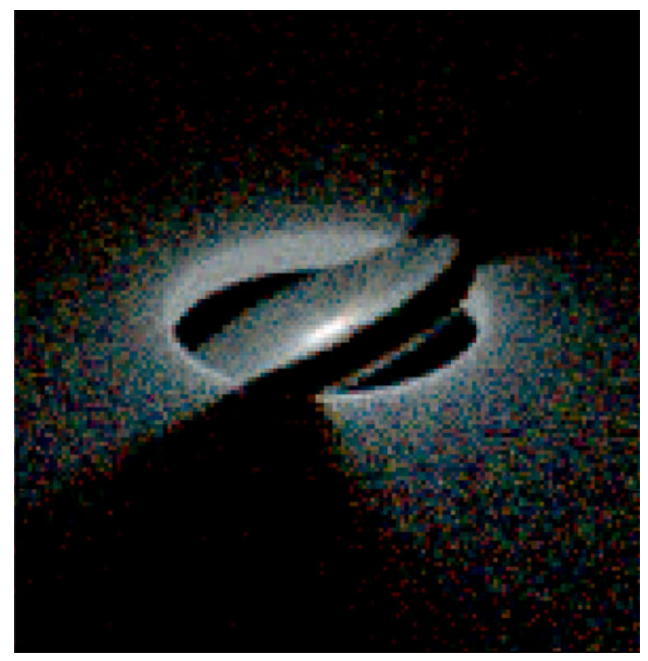

Figure 1. Figure adapted from Whitney et al. (2013, CAAS, reproduced by permission), with a three-dimensional radiative transfer prediction in $J H K$ for a warped disk configuration. The field is $4 \mathrm{AU}$ on a side.

an inclination of $\sim 21^{\circ}$ (Isella et al. 2010). Similarly, in HD 135344B (also $\sim 2 \mathrm{M}_{\odot}$ ), the VLTI+MIDI inclination is $\sim 60^{\circ}$ (Fedele et al. 2008), while $\mathrm{CO}(3-2)$ data suggests $11^{\circ}$ (Dent, Greaves, \& Coulson 2005), and IR direct imaging restricts $<20^{\circ}$ (HST+NICMOS; Grady et al. 2009).

From an observational point of view, there is a degeneracy in the line-of-sight kinematics due to variable inclination, as in a warp, and the non-Keplerian flows expected from infalling gas, as identified in TW Hya and HD 142527 by Rosenfeld et al. (2012) and Rosenfeld, Chiang, \& Andrews (2014). In TW Hya, excess CO emission at high velocities, additional to that expected from axially symmetric disk models, could be due to a warp, that may also account for the faint azimuthal modulation in scattered light imaging reported by Roberge, Weinberger, \& Malumuth (2005). In HD 142527, the infalling gas reported by Casassus et al. (2013b) and Casassus et al. (2013a) could instead be due to a warp.

\subsection{The HD 142527 warp}

The unambiguous identification of warps requires further evidence, in addition to the possible inclination trends with angular scales (which could be related to a variety of physical structures other than warps) and the hints provided by the molecular line data. Radiative transfers effects due to warps can provide such evidence. For instance, in HD 100546, Quillen (2006) recognised how a warped structure could approximate the spiral pattern seen by HST (Grady et al. 2001), thereby illustrating the potential of radiative transfer effects to account for non-axially symmetric structures. Indeed, Whitney et al. (2013) apply three-dimensional radiative transfer to warped disk architectures, as shown in 


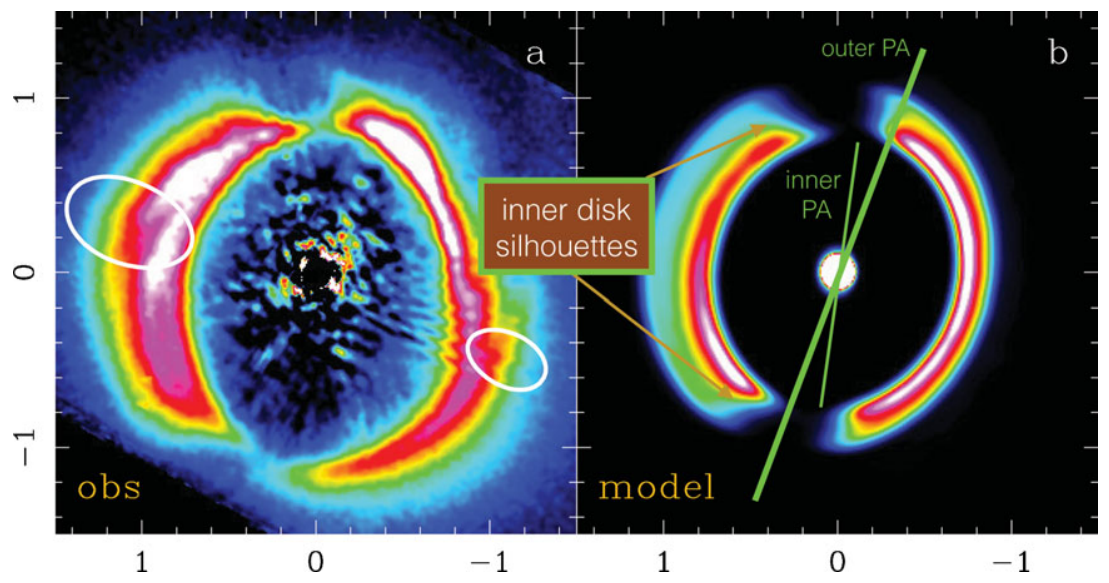

Figure 2. Comparison between the observed $H$-band polarised intensity image of HD 142527 (afrom Avenhaus et al. 2014a) and three-dimensional radiative transfer predictions (b- from Marino et al. 2015a, this is an updated version of their Figure 2). The kinematics of $\mathrm{C}^{18} \mathrm{O}(2-1)$ emission (Perez et al. 2015a) give the orientation of the outer disk; the white contours in a- correspond to systemic velocities, so that the PA of the outer disk lies at $\sim 160^{\circ}$ East of North, as indicated on b-. The inner disk shadows cast on the outer disk are best reproduced with a PA of $172^{\circ}$, the curvature of their outline (or silhouette) is reminiscent of the observations for the Eastern side. The similarities with the observations are particularly good given the idealisations of the model, which assumes a circular cavity.

Figure 1, showing that shadows are expected in the outer disks.

HD 142527 is an example of how such radiative transfer effects in scattered light can unambiguously determine the disk orientation and the existence of variable inclinations. In this case, the relative inclination change between the outer and inner disks reaches $\sim 70^{\circ} \pm 5^{\circ}$, as shown by Marino, Perez, \& Casassus (2015a). Despite such a dramatic inclination change, the intensity dips originally identified by Casassus et al. (2012) eluded interpretation for $3 \mathrm{yrs}$, because the direction connecting the dips is offset from the star, in contrast with that naively expected for a simple tilt. Yet, as illustrated in Figure 2, the silhouette of the shadows cast by the inner warp provide unequivocal evidence for this warp, which is further supported by the gas kinematics discussed in Section 3.

\subsection{Near-term prospects: how common are warps?}

Although there are indications for the frequent occurrence of inclination changes in gas-rich systems, there is so far only one clear case of a warp, i.e. in HD 142527. Next-generation adaptive-optics cameras, such as SPHERE and GPI, should soon provide substantial improvements, particularly through PDI which appears ideally suited to trace illumination effects, and so identify warps. First, results from such new AO imaging are indeed revealing intriguing intensity modulations, as in MWC 758 (Benisty et al. 2015), HD 135344B (Wahhaj et al. 2015), and in HD 100453 (Wagner et al. 2015). Follow-up molecular-line observations with Atacama Large Millimeter Array (ALMA) may provide the necessary clues from the gas kinematics.

\section{GAS IN CAVITIES}

Models predict that young giant protoplanets carve a deep gap in the dust component of protoplanetary disks, and a shallower gap in the gas and very small grains (VSGs) components (e.g. Paardekooper \& Mellema 2006; Fouchet, Gonzalez, \& Maddison 2010). The clearing of the protoplanetary gap is thought of as an important mechanism underlying the class of TDs, and residual gas in cavities has thus been intensely searched for.

This section describes observations of intra-cavity gas that select sufficiently large cavities to be resolved with current gas tracers, so with radii greater than a dozen AUs at the very least. Smaller cavities and inner disks have been detected by broad-band long-baseline optical interferometry (e.g. the gaps in HD 100546 or HD 139614 [Tatulli et al. 2011, Matter et al. 2016]). Such observations do not yet sample the intra-cavity gas (except for the central few stellar radii in Bry, e.g. Mendigutia et al.). The continuum interferometric observations have allowed to isolate the crystalline spectrum of the inner disks (as in HD 142527 [van Boekel et al. 2004]), and to study the occurrence bof gaps in HAeBes (Menu et al. 2015)

\subsection{Ro-vibrational gas and very small grains}

Long-slit spectroscopy has provided indirect evidence for gas inside cavities, under the assumption of azimuthal symmetry and Keplerian rotation (Carr, Mathieu, \& Najita 2001; Najita, Carr, \& Mathieu 2003; Acke \& van den Ancker 2006; van der Plas et al. 2008; Salyk et al. 2009). Spectro-astrometry has also been used to infer a residual gas mass from rovibrational CO emission (Pontoppidan et al. 2008; van der 


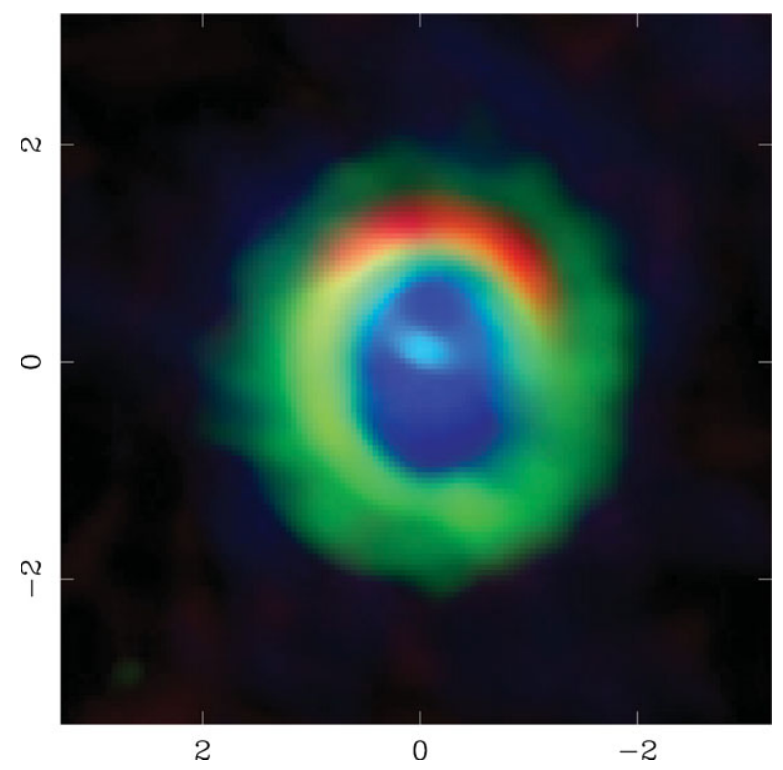

Figure 3. Summary of Cycle 0 band 7 observations, from MEM maps, with continuum in red, $\mathrm{HCO}^{+}(4-3)$ in green, and $\mathrm{CO}(3-2)$ in blue (adapted from Casassus et al. 2013b). $x$ - and $y$ - show offset from the star, in arcsec. Velocities have been restricted to highlight the fainter structures seen in $\mathrm{HCO}^{+}$, which are otherwise dwarfed by the fast $\mathrm{HCO}^{+}$central emission.

Plas et al. 2009; Pontoppidan, Blake, \& Smette 2011). A comprehensive analysis is presented in van der Plas et al. (2015) and Banzatti \& Pontoppidan (2015). The general picture is that dust cavities do indeed contain some amount of residual gas, as expected in the context of dynamical clearing.

The smallest grains are thought to be well coupled with the gas, so that they can be used as proxies. Thermal IR observations suggest the existence of smaller gaps in VSGs than in silicates, and have also led to the finding of gaps otherwise undetectable through the SED alone (Maaskant et al. 2013; Honda et al. 2015). Detailed studies of the gap structure in ro-vibrational lines along with infrared data have been performed in HD 135344B (Garufi et al. 2013; Carmona et al. 2014).

\subsection{Resolved radio observations of intra-cavity gas}

While the ro-vibrational detections pointed at residual intracavity gas, surprises were nonetheless brought by the first resolved images (Figure 3, Casassus et al. 2013b), made possible thanks to the advent of ALMA. The finest angular resolutions from ALMA Cycle 0 were just sufficient to resolve the largest TD cavity, i.e. that of the HD 142527 disk. A few $M_{\text {jup }}$ worth of gas was indeed seen inside this cavity from CO isotopologues (Perez et al. 2015a). However, the gas kinematics were puzzling, and seemed consistent with radial infall as illustrated by the fast and centrally peaked $\mathrm{HCO}^{+}(4-3)$. Slower intra-cavity $\mathrm{HCO}^{+}$signal seemed to connect with the outer disk along filamentary structures, reminiscent of protoplanetary accretion streams. But the slow intra-cavity $\mathrm{HCO}^{+}$was faint in these Cycle 0 data, and the

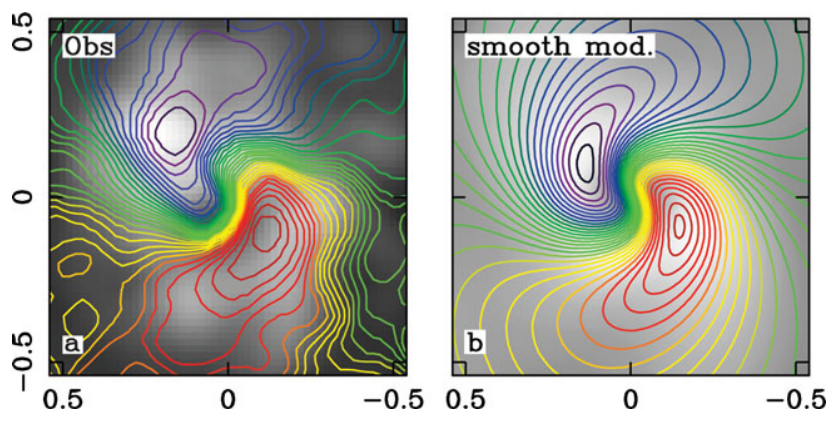

Figure 4. Figure adapted from Casassus et al. (2015a), showing the observed $\mathrm{CO}(6-5)$ kinematics in the central regions of HD 142527, and comparing with model predictions for the accretion through the warp represented in Figure 5 (convolved at the resolution of the centroid map). The origin of coordinates is set to the stellar position.

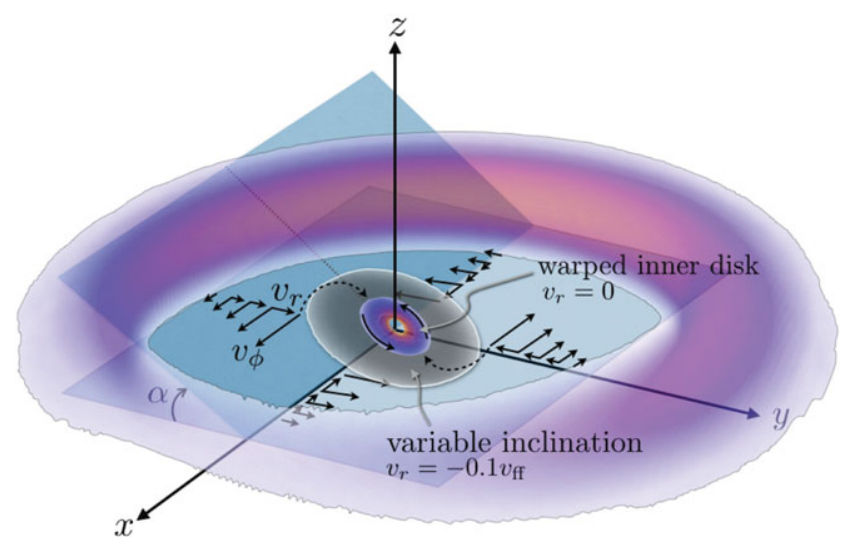

Figure 5. Sketch of the accretion kinematics through the warp in HD 142527 (from Casassus et al. 2015a). The crescent in hues of red and purple represents the distribution of mm-sized grains. Inside the warp, as it connects the two disk inclination material flows along the dashed and curved arrows.

cavity radius was sampled only with three beams. New observations in $\mathrm{HCO}^{+}(4-3)$ are required to ascertain its structure.

A more accurate view of the intra-cavity kinematics in HD 142527 is provided by the $\mathrm{CO}(6-5)$ line, with a higher frequency and a finer Cycle 0 beam. $\mathrm{CO}(6-5)$ is also free of the interstellar absorption that affects $\mathrm{CO}(3-2)$ (Casassus et al. 2013c, 2013a). Given the warped structure of HD 142527, the line-of-sight $\mathrm{CO}(6-5)$ can be understood as infall, at the observed stellar accretion rate (Garcia Lopez et al. 2006), but along and through the warp. As illustrated in Figures 4 and 5, the observations are fairly well accounted for by a model with gas inside the continuous warp. At the radius where the disk plane crosses the sky, the kinematics are Doppler-flipped so that blue turns to red, and convolution with the angular resolution results in the ' $\mathrm{S}$ '-shaped centroids. Interestingly, a fast and narrow warp improves the model, such that the two disk orientations are connected within $\sim 3 \mathrm{AU}$, at a radius of $20 \mathrm{AU}$, with material flowing orthogonal to the local disk plane at a velocity comparable to Keplerian. These observations are based on non-parametric image synthesis that pushes the limits of the Cycle 0 beam, so 
that further observations are required to ascertain the details of the gaseous flows inside the warp.

A likely origin for the observed accretion dynamics in HD 142527 probably involves the low-mass companion, at $\sim 12$ AU and with a mass ratio $\lesssim 1 / 10$ (Biller et al. 2012; Close et al. 2014; Rodigas et al. 2014). The proper-motion of the companion (Lacour et al. 2015) seems to indicate an orbit in clockwise rotation, as for the whole disk. If HD 142527B is contained in the tilted inner disk, or if it is close to it, the properties of the warp caused by the HD142527A+B system resemble disk tearing in strongly warped circumbinary disks (Nixon, King, \& Price 2013; Nealon, Price, \& Nixon 2015; Doğan et al. 2015). In the context of circumstellar disks, Facchini, Lodato, \& Price (2013) and Facchini, Ricci, \& Lodato (2014) show that in the thick-disk regime, where $\alpha<H / R$, an inner warp greater than $\sim 40^{\circ}$ will break the disk into distinct planes. Note, however, that the low mass companion at $\sim 12 \mathrm{AU}$ is unlikely to have originated the $140 \mathrm{AU}$ cavity, unless it is undergoing oscillations in inclination and eccentricity (i.e. Kozai cycles, see Martin et al. 2014). Further progress in the interpretation of the HD 142527 warp requires better data and tailored hydrodynamical models.

\subsection{Residual gas in cavities}

A summary of several other detections with ALMA of residual gas in TD cavities is given by van Dishoeck et al. (2015). Bright $\mathrm{CO}(6-5)$ emission was found ascribed to the cavities of HD 135344B and SR21 (Pérez et al. 2014), as well as in LkCa 15, RXJ 1615-3255, and SR 24S (van der Marel et al. 2015a). The size of the ring in [PZ99]J160421.7-213028 is smaller in $\mathrm{CO}(2-1)$ than in the dust continuum (Zhang et al. 2014), much as in SZ 91(Canovas et al. 2015). The depth of the cavity in mm-sized grains follows from the submillimetre continuum. Results so far are limited by dynamic range, with depths in the mm-sized dust deeper than $\sim 1 / 100$ relative to the outer disk.

The recent state of the art $345 \mathrm{GHz}$ continuum and $\mathrm{CO}(3-2)$ isotopologue data analysed by van der Marel et al. (2016) in terms of a thermochemical model, suggests that the gas cavities are up to three times smaller than the dust rings in DoAr 44, SR 21, HD 135344B, and IRS 48 (see also Bruderer et al. 2014). The drop in gas surface density can be up to $\delta \sim 10^{-2}$ (the exact values vary in each object and can be calculated from the parameters given in Table 3 of van der Marel et al. 2016).

The main result that transpires from the above studies, including HD 142527 (Perez et al. 2015a), is that the depth of the cavity is shallower in the gas than in the dust, by at least a factor of 10 . However, while van der Marel et al. (2016) investigate gradual cavity edges, angular resolution limits prevent firm conclusions on the distribution of the gas. Most studies assume axially symmetric and sharp cavity edges, parametrised by a step-function drop, with a depth $\delta \sim 1 / 10-1 / 100$ relative to the outer disk in the gaseous component. Due to the coarse linear resolutions available, the accretion kinematics remain largely unsampled. For instance, in HD 142527 sufficient linear resolution is crucial to trace the non-Keplerian accretion flows, which are otherwise much less conspicuous when sampled with a beamwidth comparable to the cavity radius (Perez et al. 2015a).

\subsection{Near-term prospects: structure and origin of the cavities}

The finest angular resolutions available from the latest ALMA results (van der Marel et al. 2016) do not yet provide enough linear resolution to sample the cavity edges. However, at the time of writing several ALMA projects are currently underway in gas-rich cavities, and the first results from the next-generation $\mathrm{AO}$ cameras are soon due. With much finer angular resolutions, it will be possible to understand better the distribution of the gas in TDs and its kinematics, and so reconcile mass transport across TD cavities with the stellar accretion rates (which has so far only been possible in HD 142527).

A key question is on the shape of the cavity edge, which is currently modelled as a step function. Yet, the edge of the cavity gives important clue on the clearing mechanism: for instance, if planet formation, the sharpness of the edge is a function of the mass of the outermost body (e.g. Crida, Morbidelli, \& Masset 2006; Mulders et al. 2013).

Intriguingly very few companions have been found in TDs (and only one candidate near the edge of a cavity, i.e. in the HD 169142 disk, see Section 6). A spectacular example is HD 142527, with a record-sized cavity of $140 \mathrm{AU}$ and no intra-cavity protoplanet detected as yet. The binary separation in HD 142527 is $\sim 10 \mathrm{AU}$ (see Section 3.2) and could only account for a $\sim 30$ AU gas-free cavity (Artymowicz \& Lubow 1994). An interesting comparison object is the GG Tau circumbinary ring, which by contrast to HD 142527 may be explained in terms of dynamical interactions with the binary provided the edge of the cavity is 'soft' in the gas (Andrews et al. 2014; Dutrey et al. 2014).

Despite the lack of detections in TD cavities, the data in HD 142527 suggest that low-mass companions can drive dramatic warps. Thus, a possible solution to the puzzle of large cavities could perhaps be found in viewing them as circumbinary disks with low mass companions undergoing Kozai oscillations, so that when the system is coplanar the binary is very eccentric (as suggested in Casassus et al. 2015a, pending tests with hydrodynamic simulations). Thus, in HD 142527 , we could be witnessing a high-inclination phase at relatively close binary separation. Definitive conclusions on the structure of this warp require finer angular resolutions and deeper sensitivities, along with new observations in the $\mathrm{HCO}^{+}$lines. Here, we are presented with another puzzle to reconcile models of disk tearing with the indications that even though the warp appears to be abrupt, gas continuously flows through it and connects the two disk inclinations.

Is there a binary lurking in all of the large TD cavities, as in HD 142527? Low mass companions into the brown-dwarf 
mass regime are difficult to detect at very close separations. The new AO cameras along with ALMA gas kinematics should soon cast light on these questions, starting with the recent detection of fairly high-mass bodies in LkCa 15 (Sallum et al. 2015, see Section 6).

\section{LOPSIDED DISKS}

Early resolved observations of TDs already suggested that the outer rings deviate from axial symmetry (in general from SMA data, Ohashi 2008; Brown et al. 2009; Andrews et al. 2011). ALMA has brought confirmation and revealed the dramatic contrast ${ }^{4}$ of the most extreme asymmetries seen in the dust continuum. To what extent do these asymmetries trace matched asymmetries in the gas? What is the efficiency of dust segregation from the gas?

\subsection{The most dramatic asymmetries}

\subsection{1. $H D 142527$}

The submillimetre continuum from HD 142527 is approximately shaped into a crescent (Ohashi 2008; Casassus et al. 2013b; Fukagawa et al. 2013) and modulated by a temperature decrement under the shadow of the inner warp (Section 2.3). It is still not entirely clear to what extend is the submillimetre continuum reflecting a similarly pronounced asymmetry in the gas. Existing observations rely on optically thick tracers of the total gas mass (including small dust grains), so cannot conclude on the underlying gas distribution. The dense-gas tracers discussed by van der Plas et al. (2014, HCN(4-3) and CS(7-6)) are affected by chemistry and seem to anti-correlate with the continuum.

Recently, the Cycle 0 data in the optically thin CO isotopologues were further analysed by Muto et al. (2015), who estimate that the contrast in the gas-phase $\mathrm{CO}$ column is about a factor of 3. While this is all the existing information available on the gas contrast, at the time of writing, its extrapolation to the total gas mass is hampered by uncertainties from grain surface chemistry: The azimuthal structure in these transitions is not a smooth crescent; their emission peaks differ from the continuum. Perhaps, the gas-phase $\mathrm{CO}$ abundance is significantly modulated in azimuth by chemistry, or a fraction of $\mathrm{CO}$ could be depleted on dust grains, especially at the location of the continuum peak (at about $11 \mathrm{~h}$ ), where continuum grey-body temperatures are $\sim 22 \mathrm{~K}$ (Figure 6 , Casassus et al. 2015d), so below freeze-out (Jørgensen et al. 2015).

The hydrodynamic simulations tend to predict contrast ratios of order $\sim 3$. Large-scale crescents in the gas surface density arise naturally in models of cavity clearing by giant planets (e.g. Zhu \& Stone 2014). Such crescents are reproduced in hydrodynamical simulations by large-scale anticyclonic vortices, which result from Rossby wave instabilities triggered at sharp radial gradients in physical conditions.

\footnotetext{
${ }^{4}$ The ratio of the intensity extrema along a ring.
}

For instance, they have also been modelled in the context of viscosity gradients (Regály et al. 2012). An apparently different model for such crescents, leading to more pronounced asymmetries in the gas, was proposed by Mittal \& Chiang (2015) based on a global disk mode in response to a stellar offset. However, as argued by Zhu \& Baruteau (2015), if such offsets result from the shift of the system centre of mass in sufficiently massive disk, the consistent incorporation of disk self-gravity dampens the contrast of an otherwise standard large-scale anticyclonic vortex. Thus in general, the gas surface density contrasts are predicted to reach moderate values, typically $\sim 3$ and perhaps up to 10 .

The azimuthal intensity contrast in HD 142527 reaches about 30, yet there is no hint so far of a similar contrast ratio either in the small grains $(\ll 0.1 \mathrm{~mm})$, nor in molecular lines, which led Casassus et al. (2013b) to suggest that the $\mathrm{mm}$-sized dust grains that originate the ALMA continuum were segregated from the gas. In other words, the dust-to-gas mass ratio could vary with azimuth. A promising mechanism to explain such segregation is the pile-up of larger grains in local pressure maxima (e.g. Weidenschilling 1977; Barge \& Sommeria 1995; Birnstiel, Dullemond, \& Pinilla 2013; Lyra \& Lin 2013), as proposed to account for the even more dramatic submillimetre continuum contrast seen in IRS 48 (see below, van der Marel et al. 2013).

An important prediction of dust trapping is that progressively larger grains ${ }^{5}$ should be more sharply confined. Multifrequency radio observations may test this prediction, since grains of progressively larger size dominate the continuum emission at correspondingly longer wavelengths. Indeed, $34 \mathrm{GHz}$ ATCA observations of HD 142527 revealed a compact clump of cm-wavelength-emitting grains buried into the ALMA crescent (Figure 6, Casassus et al. 2015d). Populations of larger grains correspond to shallower optical depth spectral index $\beta$ (e.g. Testi et al. 2014), in a parametrisation of the optical depth spectrum such that $\tau=\tau_{\circ} \times\left(\nu / \nu_{\circ}\right)^{\beta_{s}}(\beta$ is also referred to as emissivity index). Thus, three frequency points can be used to solve for the radiation temperature, optical depth, and $\beta$ index at each line of sight. Frequencies above $\sim 345 \mathrm{GHz}$ turned out to be optically thick (Figure 6, Casassus et al. 2015d), so that the spectral index trends at ALMA frequencies are due to optical depth effects rather than the underlying dust population. The ATCA clump translates into a local minimum in the $\beta$ index, indicative of larger grains.

Interestingly, the optical depth at $345 \mathrm{GHz}$ (Figure $6 \mathrm{a}$, bottom), shows an extension towards $2 \mathrm{~h}$. Yet, the minimum in opacity spectral index $\beta$ lies at $11 \mathrm{~h}$, which should be the core of the pressure maximum in the dust trap scenario. Since rotation in HD 142527 goes clockwise, a possible interpretation lies in the phenomenon predicted by Baruteau \& Zhu (2015), that larger grains should concentrate significantly ahead of the dust trap. Thus at $2 \mathrm{~h}$, the line of sight would intercept a disk-averaged grain population, modulated by the gaseous crescent, but the addition of larger grains (perhaps

\footnotetext{
${ }^{5} \mathrm{Up}$ to Stokes numbers of $\sim 1$.
} 


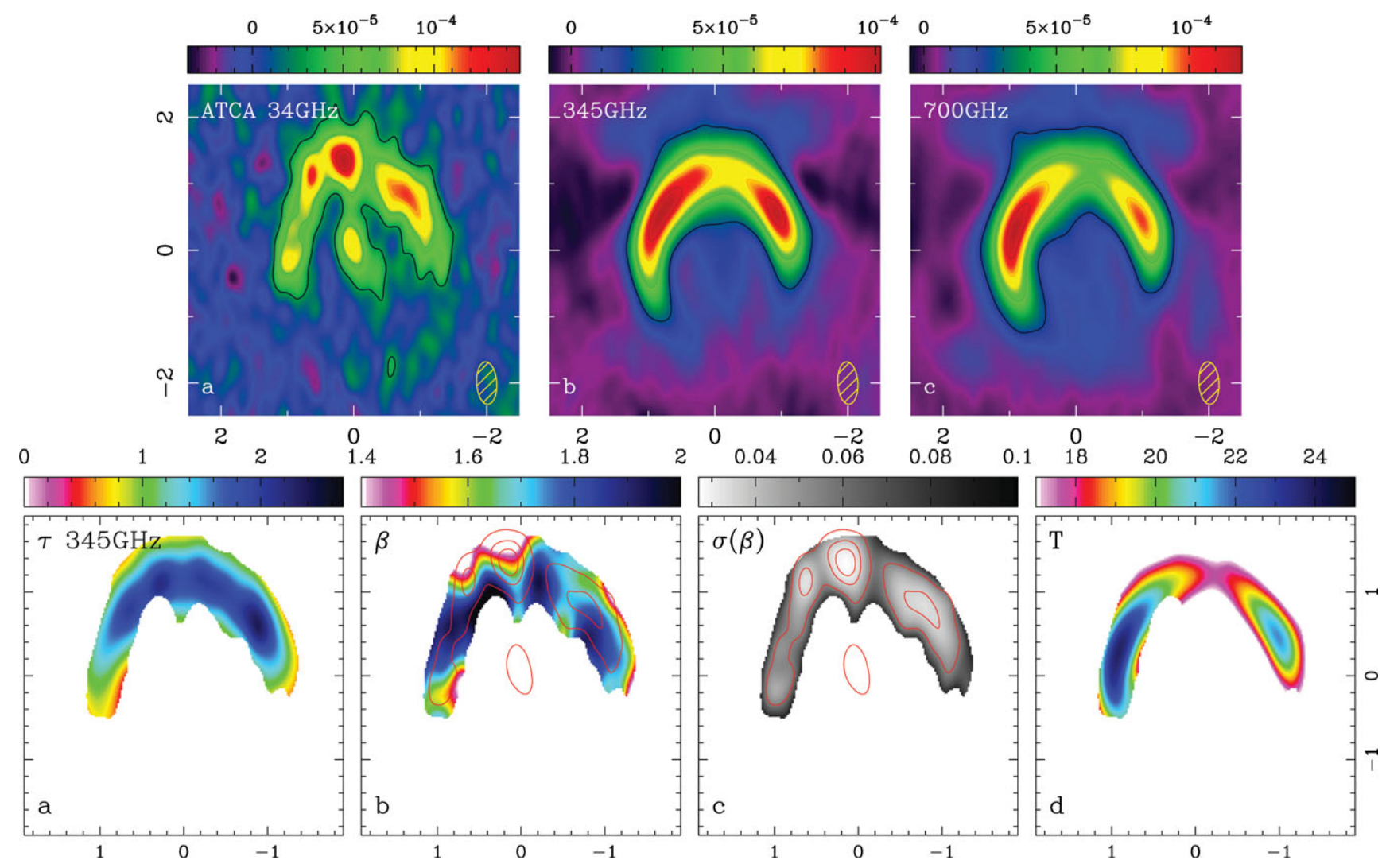

Figure 6. Figure adapted from Casassus et al. (2015d). Upper row: multi-frequency data of HD 142527 brought to a common $u v$-coverage. The ALMA data have been filtered for the ATCA response with Monte Carlo simulations of ATCA observations on deconvolved models of the ALMA data. (a): restored ATCA image at $34 \mathrm{GHz}$. (b): average of Monte Carlo (MC) simulations of ATCA observations on the ALMA band 7 data. (c): MC simulations of ATCA observations on the ALMA band 9 data. Lower row: Grey-body diagnostics inferred from the multi-frequency data. (a): optical depth map at the reference frequency of $345 \mathrm{GHz}$. (b): line of sight emissivity index map $\beta$, with ATCA specific intensity contours in red. (c): root-mean-square uncertainties on the emissivity index map. (d): line of sight temperature, $T_{s}$.

up to $1 \mathrm{~cm}$ sizes in this disk), might raise the total optical depth even away from the pressure maximum. However, such a shift of the larger grains is possible only with Stokes numbers $S \gtrsim 1$ (Baruteau \& Zhu 2015), which for 1 cm grains would require a disk mass $\sim 100$ times lower than inferred from the SED modelling (Casassus et al. 2015d), and/or a correspondingly low gas-to-dust mass ratio.

Thus, the bulk of the data so far indicates that the spatial segregation of dust sizes in azimuth is indeed at work in the lopsided outer ring of HD 142527. However, while models have been proposed that approximate the observed segregation (through parametric models, Casassus et al. 2015d), they are fine-tuned in key parameters such as the $\alpha$ turbulence prescription and the threshold grain size for trapping.

\subsubsection{IRS 48}

The record-holder lopsided TD is IRS 48, where van der Marel et al. (2013) found that the contrast in ALMA band 9 is greater than 100 , so three times as pronounced as in HD 142527. They reproduce such dramatic contrast with their dust trapping prescriptions.

Observations of IRS 48 are hampered by intervening cloud emission from $\rho \mathrm{Oph}$, with $A_{\mathrm{V}} \sim 10$. Yet, by using the rarer $\mathrm{CO}$ isotopologues at higher velocities, Bruderer et al. (2014) estimated that the total gas mass of the disk is only $\sim 10^{-4} \mathrm{M}_{\odot}$.

The dramatic contrast observed in IRS 48 would seem very unlikely to correspond to an equally lopsided gas distribution, and is thus probably due to segregation of grain sizes. Indeed, van der Marel et al. (2015b) report on the tentative detection of the corresponding spectral trends in a comparison between VLA and ALMA observations. The confirmation of lopsidedness at optically thin VLA frequencies (see Figure 7) dissipates worries that the asymmetry observed at high frequencies, which are likely optically thick, could be related to optical depth effects in this highly inclined disk.

\subsection{3. $M W C 758$}

Can all continuum asymmetries in TDs be interpreted as dust traps? MWC 758 presents an interesting caveat. The VLA $34 \mathrm{GHz}$ image presented by Marino et al. (2015b, reproduced in Figure 8), when compared with the more extended signal seen by ALMA, seems fairly consistent with the dust trap scenario, at least as well as in HD 142527 or IRS 48 given the available constraints. But in fact two pressure maxima (or anticyclonic vortices) would seem to be 


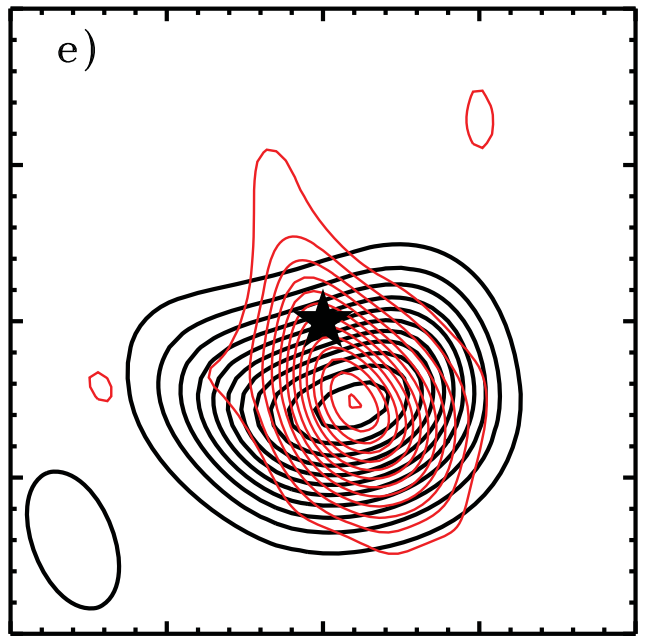

Figure 7. Figure adapted from van der Marel et al. (2015b, their Figure 1, (C)AAS, reproduced with permission). VLA observations of IRS 48 at optically thin frequencies confirm the extreme lopsidedness of this TD. VLA observations at $34 \mathrm{GHz}$ are shown in red contours-the tail towards the NE is likely due to stellar emission. ALMA band 9 observations at $680 \mathrm{GHz}$ are shown in black contours, after filtering for the VLA response. The field is $2 \operatorname{arcsec}$ on a side. While at $34 \mathrm{GHz}$ the crescent appears to be somewhat more compact, the role of optical depth effects in widening the $680 \mathrm{GHz}$ signal remain to be quantified.

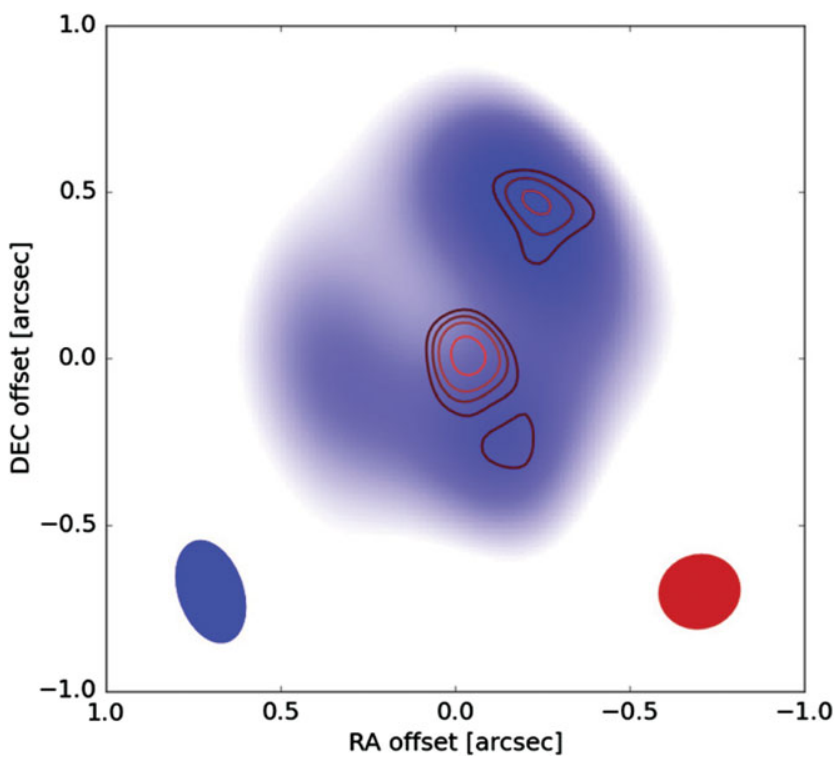

Figure 8. Figure adapted from Marino et al. (2015b, their Figure 3). VLA observations of MWC 758 compared to a deconvolved model of the ALMA band 7 visibilities. The VLA signal is shown in red contours-the red ellipse corresponds to the synthesised beam. The blue scale corresponds to an 'MEM' non-parametric model of the $337 \mathrm{GHz}$ ALMA data-the blue ellipse corresponds to an elliptical Gaussian fit to the point-spread-function of the 'MEM' algorithm.

required to account for the double-peaked ALMA signal in MWC 758. Besides estimates of the submillimetre optical depth are missing - which requires finer angular resolution submillimetre data, as well as an additional frequency point intermediate with the VLA.
The compact VLA signal in MWC 758 may also be looked at from an entirely different perspective. Another interpretation could follow from the recent proposal by Dong et al. (2015a) that the observed spiral pattern in MWC 758 are launched by planet could be launched by a planet exterior to the arms, at a radius of $\sim 0.6$ arcsec. Marino et al. (2015b) caution that the observed signal is quite close to the location of such a body, as illustrated in Figure 9. Thus, the compact signal could be circumplanetary dust that is heated by planet formation feedback, (as in the positive feedback leading to enhanced viscous heating proposed by Montesinos et al. 2015).

\subsection{Mild asymmetries and radial dust traps}

The above examples for extreme submillimetre continuum asymmetries are seen in few $\mathrm{HAeBe}$ stars (stellar mass $2 \mathrm{M}_{\odot}$ ), and are not typical of the average TD. Most TDs show milder azimuthal asymmetries when resolved in the submillimetre continuum. Examples of mild contrast ratios, $\lesssim 4$ or as could be reached by the gas, are HD 135344B, SR 21 (Pérez et al. 2014; Pinilla et al. 2015), and $\mathrm{LkH} \alpha 330$ (Isella et al. 2013). Given their coarseness, these observations could accommodate sharper asymmetries.

An interesting avenue to explain such mild asymmetries could be spiral arm crowding. Indeed the asymmetry reported in HD 135344B (Pérez et al. 2014) with a relatively coarse beam in ALMA Cycle 0, has been resolved into a convergence of spiral arms (van der Marel et al. 2016, their Figure 1). These spirals were hinted at in axisymmetric model residuals (Pérez et al. 2014; Pinilla et al. 2015). Local shock heating from the spiral waves (Rafikov 2016) could perhaps further pronounce asymmetries related to spirals, and lead to mild asymmetries when observed in a coarse beam.

There are also examples of axially symmetric TDs, such as the TTauri J160421, SZ 91, DoAr 44 (Zhang et al. 2014; van der Marel et al. 2016), and SZ 91 (Canovas et al. 2016). While azimuthal dust segregation or trapping does not seem to be required in such systems with no or mild asymmetries, on the other hand, the radial segregation of dust grain sizes seems to be systematically present in TDs. Radial trapping is often invoked as a means to halt the catastrophic inward migration of grains due to aerodynamic drag (Weidenschilling 1977). The smaller and shallower cavities in the gas phase compared to the dust were introduced in Section 3 as a feature of dynamical clearing by giant planet formation. This is the same phenomenon as radial trapping, since it is the radial bump in gas pressure generated by planet formation that traps the larger dust grains in the outer disk (e.g. Pinilla, Benisty, \& Birnstiel 2012; Pinilla et al. 2015).

\subsection{Near-term prospects: the gas background and vortex velocity fields}

The dust trap phenomenon is clearly at work in the two most extreme asymmetries, and could be an important mechanism 

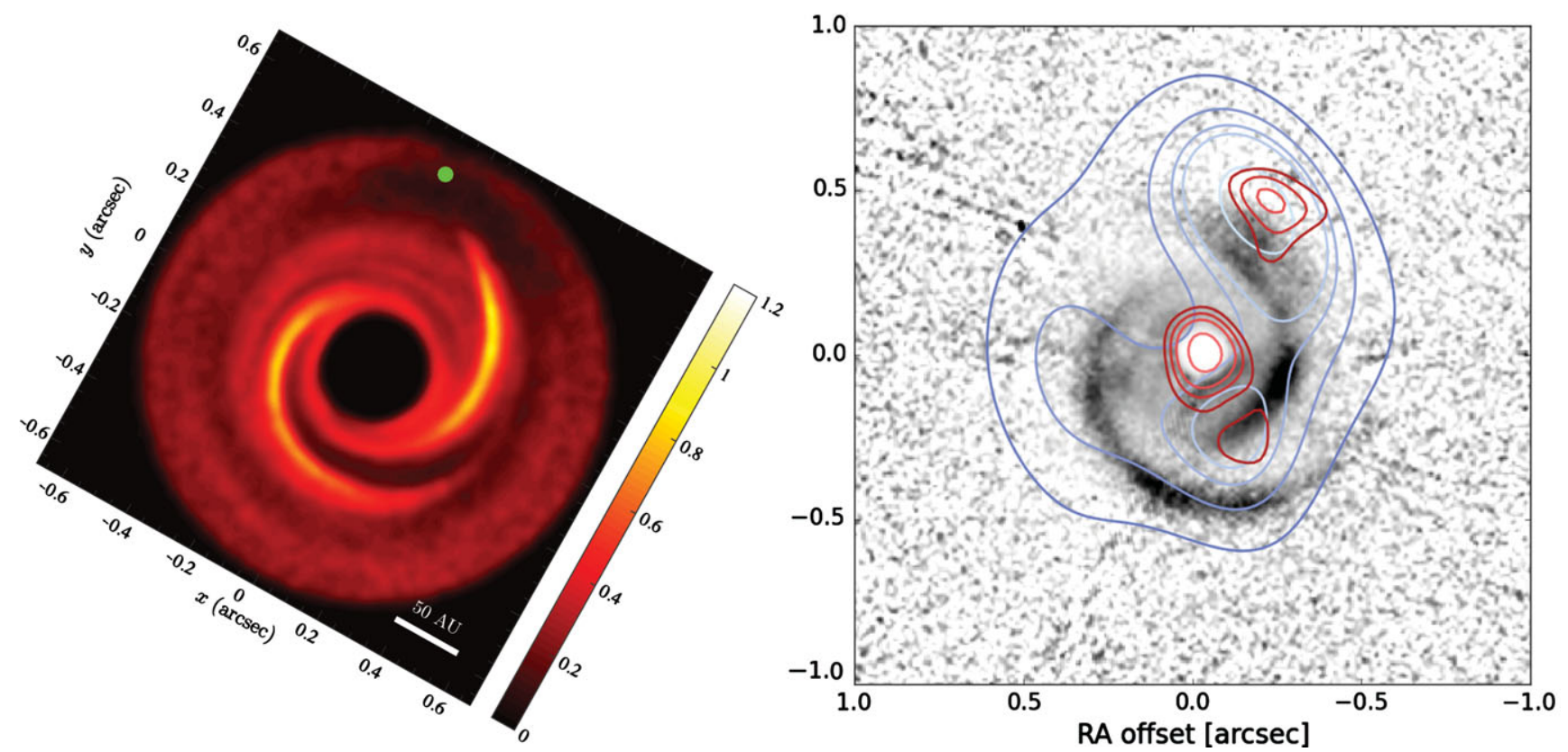

Figure 9. Left: Spiral model involving a planetary mass companion, from Dong et al. (2015a, part of their Fig. 4, (CAAS, reproduced with permission). The companion is highlighted as a green dot-and the field is rotated so that the companion approximately matches the location of the VLA clump seen in MWC 758. Right (adapted from Marino et al. 2015b): VLA data, in red contours, and ALMA 345GHz, in blue contours, overlaid on the SPHERE $Y$-band polarised intensity image (Benisty et al. 2015), in grey scale.

in the evolution of the dust grain population. However, accurate knowledge of the gas background and physical conditions is required to estimate the efficiency of trapping by aerodynamic coupling. As yet, there has been no confirmation of the velocity fields expected in large-scale vortices, so an important unknown on the origin of the lopsided gas distributions remains untackled. Another open question is, for instance, the impact of enhanced cooling in the dust trap on the maximum possible gas density contrast.

Taking the dust trapping scenario one step further poses a question on the potential of such dust traps to form gaseous giants. What would be the impact on the disk of massive bodies forming inside a dust trap at large stellocentric distances? Are there signs of such bodies?

Both the observational and theoretical situations are quickly evolving. ALMA should soon provide additional multi-frequency continuum data with which to better quantify the degree of trapping and the corresponding grain distributions. Perhaps, resolved molecular line maps of dust traps could be used to detect the expected vortex velocity fields. Without an observational confirmation, however, the question on the origin of the gaseous lopsided structures is still open.

\section{SPIRALS}

Observations in optical/IR scattered light of spirals in protoplanetary disks have been discussed on several occasions, both in discovery articles (see below) and in modelling efforts (Juhász et al. 2015; Pohl et al. 2015; Dong et al. 2015b). The single-star systems where spirals have been reported seem to be TDs, i.e. they host either central cavities or gaps. It is possible that these systems are in fact binaries with a low mass ratio: their spiral patterns bear similarities with the arclike features seen in some binary disks, such as in AS 205 (Salyk et al. 2014) and SR 24 (Mayama et al. 2010). The following is a list of spiral patterns in disks around primary stars that dominate by mass (relative to possible low mass bodies embedded in the disks), some of which are shown in Figure 10:

- HD 100546, with a 13 AU gap (e.g. Tatulli et al. 2011; Avenhaus et al. 2014b), and tightly wound and complex spirals seen in the outer disk (Grady et al. 2001; Boccaletti et al. 2013);

- HD 142527, with a 140 AU gap and both a grand design spiral pattern in the outer disk as well as more intricate spiral features carving the outer edge of the cavity (Fukagawa et al. 2006; Rameau et al. 2012; Casassus et al. 2012; Canovas et al. 2013; Avenhaus et al. 2014a);

- AB Aur, with a gap between 40 and $140 \mathrm{AU}$ (Hashimoto et al. 2011), also exhibits large scale spirals that modulate the outer disk, and seen in scattered light (Grady et al. 1999; Fukagawa et al. 2004);

- HD 141569A, in the transition to debris disk (e.g. Thi et al. 2014), with several tightly-wound concentric rings or spirals extending out to $400 \mathrm{AU}$, and a $175 \mathrm{AU}$ cavity (Biller et al. 2015), also hosts large-scale open spirals that may result from interaction with the other members of this hierarchical multiple system (Clampin et al. 2003); 
AB Aur

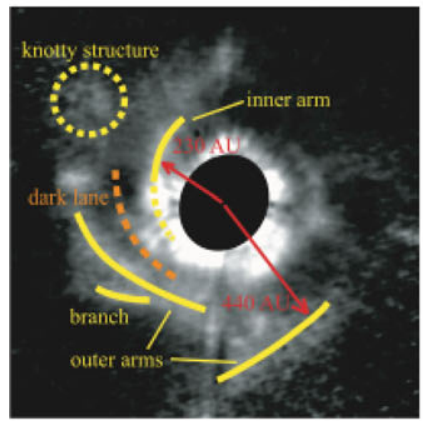

HD 141569A

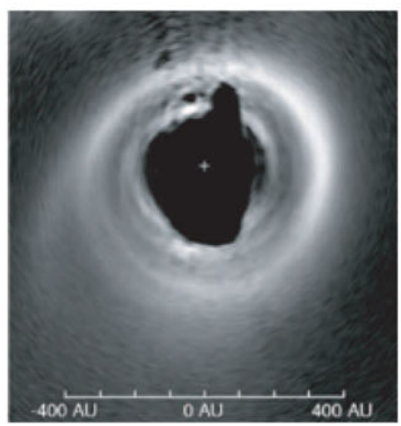

HD 135344B

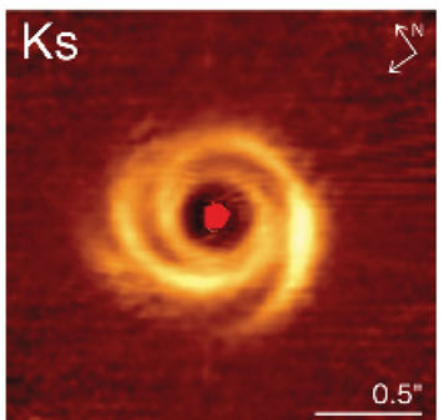

HD 100453

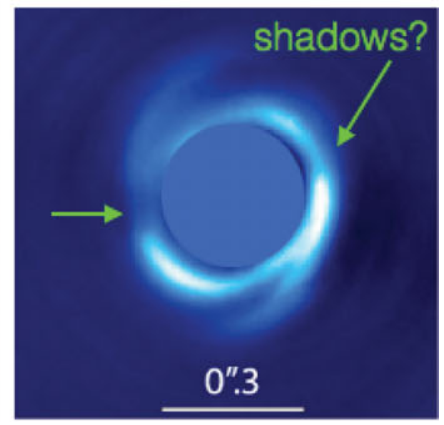

Figure 10. Gallery of optical/IR spirals. From left to right, we show scattered-light images of AB Aur (Fukagawa et al. 2004, part of their Fig. 3, CAAS, reproduced with permission), HD 141569A (Clampin et al. 2003, part of their Fig. 8, CAAS, reproduced with permission), HD 135344B (Garufi et al. 2013, part of their Fig. 1, CESO, reproduced with permission), and HD 100453 (Wagner et al. 2015, part of their Fig. 2, CAAS, reproduced with permission). All figures have been reproduced by permission of the AAS or A\&A. In HD 100453, we have highlighted two intensity dips where the two-armed spiral pattern seem to stem from. These dips are very reminiscent of the HD 142527 shadows, which also seem to be at the root of spirals.

- MWC 758, with a central 100 AU cavity detected in the submillimetre (Isella et al. 2010) but not in the near-IR (Grady et al. 2013), shows a large-scale grand design two-armed spiral (Grady et al. 2013; Benisty et al. 2015) recently interpreted as interior spirals triggered by a companion at fairly wide separation $(0.5 \mathrm{arcsec}$, see Figure 9, Dong et al. 2015a);

- HD 135344B has a 30 AU dust cavity (Carmona et al. 2014), and a two-armed grand-design spiral best seen with PDI (Muto et al. 2012; Garufi et al. 2013; Wahhaj et al. 2015);

- HD 100453 is a recent addition to the list of known spirals in TDs (Wagner et al. 2015; Dong et al. 2016). This HAe disk, with ring enclosing a cavity of $\sim 20 \mathrm{AU}$, displays a two-armed spiral pattern stemming from the ring, fairly open and extending out to $\sim 42 \mathrm{AU}$.

There are, however, only two examples of molecular-line counterparts to the near-IR spirals in protoplanetary disks. The AB Aur IR spirals were also detected in the $\mathrm{CO}$ isotopologues (Corder, Eisner, \& Sargent 2005; Lin et al. 2006). Intriguingly, the spirals in AB Aur appear to counterrotate with the disk, which led Tang et al. (2012) to propose that they stem from an infalling envelope (but the apparent counter-rotation could also result from a Doppler flip due to a warp, see Section 2.2). As summarised in Figure 11, Christiaens et al. (2014) reported very large scale ${ }^{12} \mathrm{CO}$ spirals in HD 142527, one of which appears to have a clear counterpart in the most conspicuous IR spiral found by Fukagawa et al. (2006).

The radio detection of spirals in molecular-lines is very important to estimate physical conditions, and search for kinematic trends, such as deviations from Keplerian rotation (as in AB Aur), that may help understand the origin of the spirals. Velocity dispersion maps may perhaps also be used to detect the compression wave. For now, the molecular-line spirals in HD 142527 allow estimates of temperature and column densities. Christiaens et al. (2014) find that the spirals are surprisingly cold, with $25 \mathrm{~K}$ near the continuum ring, and down to $10-15 \mathrm{~K}$ further out. The Toomre $\mathrm{Q}$ parameter, $Q=c_{\mathrm{s}} \Omega /(\pi G \Sigma)$, for each of the two spirals $S_{1}$ and $S_{2}$, are $100<Q_{S 1}<50000$, and $50<Q_{S 2}<35000$, where the lower bound stems from the continuum non-detection (assuming a gas-to-dust ratio of 100), and the upper bound is set by the assumption of optically thin ${ }^{12} \mathrm{CO}$. Thus, the spirals seen in the outer disk of HD 142527 appear to be gravitationally stable.

\subsection{Near-term prospects: radio counterparts}

The near future should see a multiplication of radio counterparts to the IR spirals (e.g. the continuum counterparts in HD 135344B; van der Marel et al. 2016). Radio observations, especially in molecular lines, are required to estimate physical conditions such as density and temperature, and so find clues as to the origin of spirals. In addition, radio observations should help in disentangling radiative transfer effects, such as proposed by Quillen (2006).

There are several on-going efforts on the theoretical side, with the coupling of three-dimensional hydrodynamics and three-dimensional radiative transfer applied to planet-disk interaction (e.g. Ober et al. 2015; Perez et al. 2015b; Dong et al. 2015a). However, in addition to the gravitational interaction with massive bodies, either planetary-mass or stellar, as in multiple stellar systems, perhaps other physical mechanisms may also launch spirals in circumstellar disks. One possibility is gravitational instability of the disk, whose observability in scattered light has recently been predicted from three-dimensional radiative transfer and hydrodynamics (Dong et al. 2015b). Another example, motivated by the obvious link of the HD 142527 spirals with the shadows cast on the outer disk by the inner warp (which may also be observed in HD 100453), is the proposal by Montesinos et al. (2016) that spirals could result from the forcing 


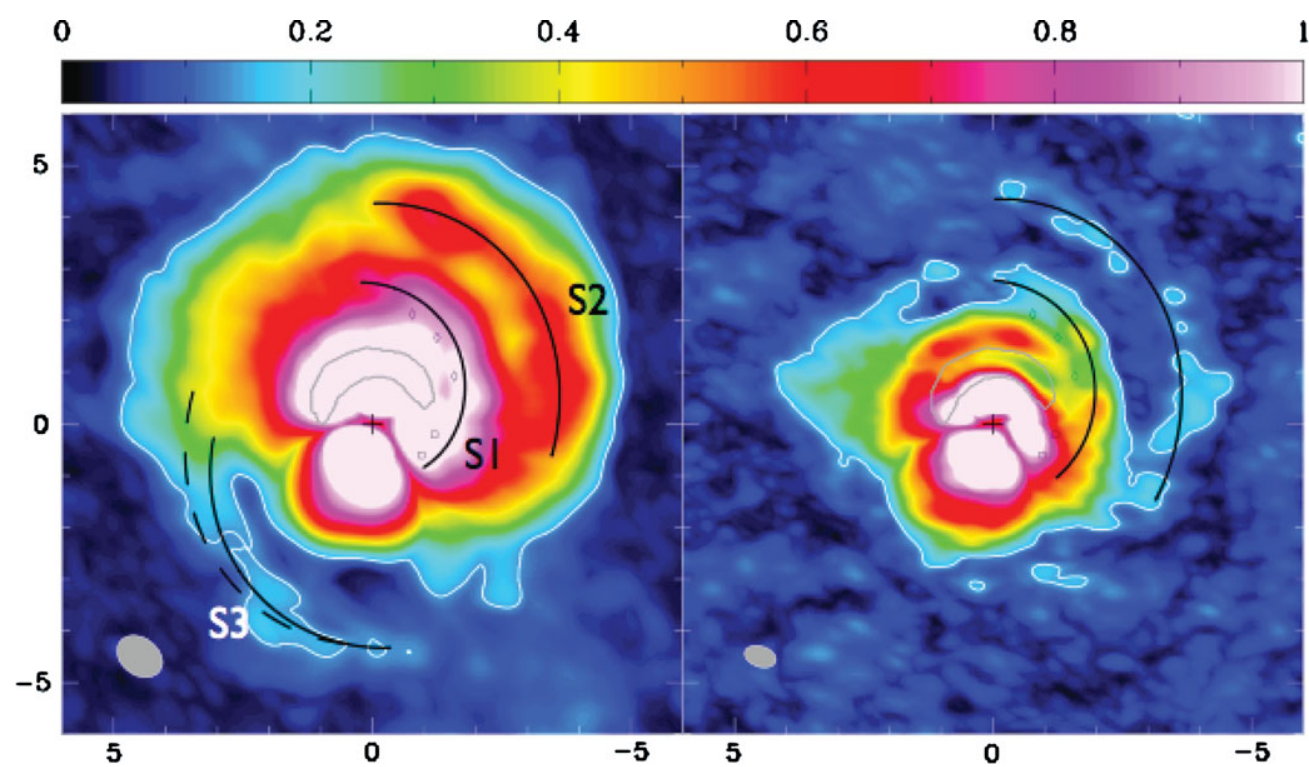

Figure 11. Radio spirals from HD 142527 (Figure adapted from Christiaens et al. 2014). The CO(2-1) peak signal is shown on the left, while $\mathrm{CO}(3-2)$ is shown on the right (the gray ellipse correspond to the clean beams). $x-$ and $y$-axis indicate angular offset from the star, along RA and Dec., and in arcsec. Since the distance to HD 142527 is about $140 \mathrm{pc}$, the spirals are seen to extend out to $700 \mathrm{pc}$ in radius. The lozenge symbols indicate the most conspicuous IR spiral, from Fukagawa et al. (2006). The inverted-V decrement in peak CO intensity, seen in both transitions, is due to interstellar absorption at velocities that correspond to these locations in the Keplerian outer disk.

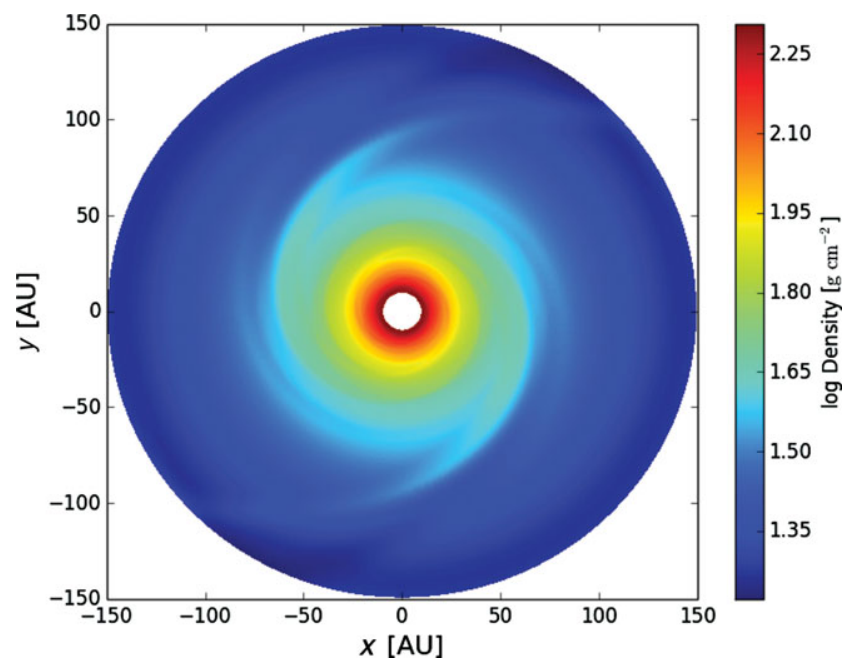

Figure 12. Spirals launched from the temperature forcing of the outer disk by shadows projected from a tilted inner disk-as in HD 142527 (and perhaps also in HD 100453). The image shows a snapshot of the density field at 250 orbits, under the effect of shadows aligned in the East-West direction (from Montesinos et al. 2016).

of the temperature field in the outer disk, as it is modulated by variable illumination due to non-axially symmetric shadowing from the inner regions. This idea is illustrated in Figure 12.

\section{THE ROLE OF EMBEDDED PROTOPLANETS}

Key to understanding the astrophysics of circumstellar disks, and eventually giant planet formation, is definitive information on the protoplanetary systems that these disks potentially host. Three promising protoplanet detections have recently been reported from their thermal signal at $\sim 3 \mu \mathrm{m}$, in the gapped disks HD 100546 (Quanz et al. 2013, 2015), HD 169142 (Reggiani et al. 2014) and in LkCa 15 (Sallum et al. 2015). In the case of LkCa 15, the interpretation in terms of an accreting body is strengthened by the detection of coincident and unresolved $\mathrm{H} \alpha$ emission.

The interpretation of unresolved continuum signals at low contrasts is difficult, as the protoplanet accretion luminosity leads to a positive feedback in stellocentric accretion (Montesinos et al. 2015), resulting in an extended thermal signal well in excess of genuine photospheric emission, and perhaps also a local enhancement in scale height. An example of such extended near-IR emission, both in thermal emission (e.g. in L band) and in scattered-light (e.g. in K band), could be the spatially extended signal from HD 100546b (Garufi et al. 2016).

In general the morphological details of the extended signal from such candidate circumplanetary regions are very difficult to ascertain because of observational biases, either in the high-contrast techniques or in near-IR interferometry, which are being tackled by technological breakthroughs (i.e. with extreme AO cameras such as GPI and SPHERE, and the next-generation interferometry instruments such as VLTI-MATISSE). Examples of such biases are the radiative 
transfer effects at finite inclination that lead to asymmetries in otherwise axially symmetric disks, and to similar closure phase signals as binary models (as in FL Cha and T Cha, Cieza et al. 2013; Olofsson et al. 2013).

A different avenue to detect embedded protoplanets, and estimate their mass, is to identify the corresponding kinematical signature of the circumplanetary disk (CPD). Perez et al. (2015b) study the observability of such CPDs with ALMA using molecular lines, by coupling 3D hydrodynamical simulations and radiative transfer, and after filtering for the instrumental response ${ }^{6}$. They predict that the CPD from an accreting gaseous giant should stand out in velocity dispersion maps. In addition the channel maps with disk emission at the position of the protoplanet should show a kink (i.e. a tight local bend), whose amplitude depends on the planet mass. Starting from ALMA Cycle 3, such long baseline observations with ALMA are routinely possible, so that prospects are good for the detection of the kinematical signatures of CPDs.

\section{CONCLUSION}

While accurate knowledge on the existence of embedded protoplanetary systems is still far from systematically available, resolved observations of TDs reveal surprising structures. The observed phenomena do not readily fit in the picture expected for TDs as sculptures of planet formation. Instead, the basic idea of flat protoplanetary disk system is being challenged by the observations of warps, whose frequency remains to be assessed. Residual gas inside cavities is clearly observed, as expected for dynamical clearing, but the concomitant protoplanetary streamers are still elusive. The observed intra-cavity stellocentric accretion, in the single case where it has been detected, occurs at much higher velocities than expected for planetary accretion streams.

The outer regions of TDs often seem to be non-axially symmetric - quite dramatically so in a couple of instances. This observation could bring confirmation for the scenario of 'dust trapping' as a way to circumvent the catastrophic infall of the larger dust grains due to aerodynamical drag. While dust size segregation has clearly been demonstrated to exist, the origin of the required gaseous asymmetry in a large anticyclonic vortex remains to be confirmed observationally. There does not seem to be a clear observational connection between the outer disk asymmetry and on-going planet formation, as illustred by the case of $\mathrm{LkCa} 15$, with a detection of protoplanetary accretion (Sallum et al. 2015), and a seemingly perfectly symmetric outer disk (van der Marel et al. 2015a).

The observations of TDs have also revealed spectacular spiral arms, the origin of which still remains the subject of intense debate. They could be launched by planets, or result from gravitational instability, or be induced from variable

${ }^{6}$ Including real phase noise taken from the HL Tau dataset presented by ALMA Partnership et al. (2015). illumination (Montesinos et al. 2016). Once launched, the frequent observation of spirals in systems with cavities and non-axially symmetric outer disks suggests that spirals may play a crucial role in disk evolution, as argued by Rafikov (2016). New data will soon provide estimates of physical conditions with which to test these ideas.

Finally, it appears that physical processes other than planet formation are governing the TD phase of Class II YSOs, since most large cavities seem empty of massive bodies. Perhaps, well identifiable planet formation structures have to be searched for at earlier evolutionary stages, i.e. in Class I disks such as HL Tau (ALMA Partnership et al. 2015; Testi et al. 2015; Dipierro et al. 2015; Gonzalez et al. 2015; Pinte et al. 2016).

\section{ACKNOWLEDGEMENTS}

We thank the following disk workers for very useful input: Henning Avenhaus, Amelia Bayo, Mark Booth, Valentin Christiaens, Lucas Cieza, Sebastián Marino, Matías Montesinos, Johan Olofsson, Sebastián Pérez, Dave Principe, Nienke van der Marel, Gerrit van der Plas and Matthias Schreiber. Financial support was provided by Millennium Nucleus RC130007 (Chilean Ministry of Economy), and additionally by FONDECYT grant 1130949.

\section{REFERENCES}

Acke, B., \& van den Ancker, M. E. 2006, A\&A, 449, 267

Alencar, S. H. P., et al. 2010, A\&A, 519, A88

ALMA Partnership, et al. 2015, ApJ, 808, L3

Andrews, S. M., Wilner, D. J., Espaillat, C., Hughes, A. M., Dullemond, C. P., McClure, M. K., Qi, C., \& Brown, J. M. 2011, ApJ, 732, 42

Andrews, S. M., et al. 2014, ApJ, 787, 148

Artymowicz, P., \& Lubow, S. H. 1994, ApJ, 421, 651

Avenhaus, H., Quanz, S. P., Meyer, M. R., Brittain, S. D., Carr J. S., \& Najita, J. R. 2014b, ApJ, 790, 56

Avenhaus, H., Quanz, S. P., Schmid, H. M., Meyer, M. R., Garufi, A., Wolf, S., \& Dominik, C. 2014a, ApJ, 781, 87

Banzatti, A., \& Pontoppidan, K. M. 2015, ApJ, 809, 167

Barge, P., \& Sommeria, J. 1995, A\&A, 295, L1

Baruteau, C., \& Zhu, Z. 2015, arXiv:1511.03498

Benisty, M., et al. 2015, A\&A, 578, L6

Biller, B., et al. 2012, ApJ, 753, L38

Biller, B. A., et al. 2015, MNRAS, 450, 4446

Birnstiel, T., Dullemond, C. P., \& Pinilla, P. 2013, A\&A, 550, L8

Boccaletti, A., Pantin, E., Lagrange, A.-M., Augereau, J.-C., Meheut H., \& Quanz, S. P. 2013, A\&A, 560, A20

Boccaletti, A., et al. 2015, Nature, 526, 230

Bouvier, J., Grankin, K., Ellerbroek, L. E., Bouy, H., \& Barrado, D. 2013, A\&A, 557, A77

Brown, J. M., Blake, G. A., Qi, C., Dullemond, C. P., Wilner, D. J., \& Williams, J. P. 2009, ApJ, 704, 496

Bruderer, S., van der Marel, N., van Dishoeck, E. F., \& van Kempen T. A. 2014, A\&A, 562, A26

Canovas, H., Ménard, F., Hales, A., Jordán, A., Schreiber M. R., Casassus, S., Gledhill, T. M., \& Pinte, C. 2013, A\&A, 556, A123 
Canovas, H., Caceres, C., Schreiber, M. R., Hardy, A., Cieza, L., Ménard, F., \& Hales, A. 2016, MNRAS, submitted

Canovas, H., et al. 2015, ApJ, 805, 21

Carmona, A., et al. 2014, A\&A, 567, A51

Carr, J. S., Mathieu, R. D., \& Najita, J. R. 2001, ApJ, 551, 454

Casassus, S., Perez, M.S., Jordán, A., Ménard, F., Cuadra, J., Schreiber, M. R., Hales, A. S., \& Ercolano, B. 2012, ApJ, 754, L31

Casassus, S., Perez, S. M., van der Plas, G., Dent, W. R. F., Hales A., \& Ménard, F. 2013a, "Transformational Science with ALMA: From Dust to Rocks to Planets", NRAO workshop (Waikoloa, Hawaii, April 8-12)

Casassus, S., et al. 2015a, ApJ, 811, 92

Casassus, S., et al. 2013b, Nature, 493, 191

Casassus, S., et al. 2013c, A\&A, 553, A64

Casassus, S., et al. 2015d, ApJ, 812, 126

Christiaens, V., Casassus, S., Perez, S., van der Plas, G., \& Ménard, F. 2014, ApJ, 785, L12

Cieza, L. A., et al. 2013, ApJ, 762, L12

Cieza, L. A., Schreiber, M. R., Romero, G. A., Williams, J. P., Rebassa-Mansergas, A., \& Merín, B. 2012, ApJ, 750, 157

Cieza, L., Williams, J., Kourkchi, E., Andrews, S., Casassus, S., Graves, S., \& Schreiber, M. R. 2015, MNRAS, 454, 1909

Clampin, M., et al. 2003, AJ, 126, 385

Close, L. M., et al. 2014, ApJ, 781, L30

Corder, S., Eisner, J., \& Sargent, A. 2005, ApJ, 622, L133

Crida, A., Morbidelli, A., \& Masset, F. 2006, Icarus, 181, 587

Dent, W. R. F., Greaves, J. S., \& Coulson, I. M. 2005, MNRAS, 359, 663

Dipierro, G., Price, D., Laibe, G., Hirsh, K., Cerioli, A., \& Lodato G. 2015, MNRAS, 453, L73

Doğan, S., Nixon, C., King, A., \& Price, D. J. 2015, MNRAS, 449, 1251

Dong, R., Hall, C., Rice, K., \& Chiang, E. 2015b, ApJ, 812, L32

Dong, R., Zhu, Z., Fung, J., Rafikov, R., Chiang, E., \& Wagner, K. 2016, ApJ, 816, L12

Dong, R., Zhu, Z., Rafikov, R. R., \& Stone, J. M. 2015a, ApJ, 809, L5

Dunham, M. M., et al. 2015, ApJS, 220, 11

Dutrey, A., et al. 2014, Nature, 514, 600

Eisner, J. A., Lane, B. F., Hillenbrand, L. A., Akeson, R. L., \& Sargent, A. I. 2004, ApJ, 613, 1049

Esau, C. F., Harries, T. J., \& Bouvier, J. 2014, MNRAS, 443, 1022

Espaillat, C., Calvet, N., D’Alessio, P., Hernández, J., Qi, C., Hartmann, L., Furlan, E., \& Watson, D. M. 2007, ApJ, 670, L135

Facchini, S., Lodato, G., \& Price, D. J. 2013, MNRAS, 433, 2142

Facchini, S., Ricci, L., \& Lodato, G. 2014, MNRAS, 442, 3700

Fedele, D., et al. 2008, A\&A, 491, 809

Fouchet, L., Gonzalez, J.-F., \& Maddison, S. T. 2010, A\&A, 518, A16

Fukagawa, M., Tamura, M., Itoh, Y., Kudo, T., Imaeda, Y., Oasa, Y., Hayashi, S. S., \& Hayashi, M. 2006, ApJ, 636, L153

Fukagawa, M., et al. 2004, ApJ, 605, L53

Fukagawa, M., et al. 2013, PASJ, 65, L14

Garcia Lopez, R., Natta, A., Testi, L., \& Habart, E. 2006, A\&A, 459,837

Garufi, A., et al. 2013, A\&A, 560, A105

Garufi, A., et al. 2016, A\&A, accepted

Golimowski, D. A., et al. 2006, AJ, 131, 3109
Gonzalez, J.-F., Laibe, G., Maddison, S. T., Pinte, C., \& Ménard F. 2015, MNRAS, 454, L36

Grady, C. A., Woodgate, B., Bruhweiler, F. C., Boggess, A., Plait, P., Lindler, D. J., Clampin, M., \& Kalas, P. 1999, ApJ, 523, L151

Grady, C. A., et al. 2001, AJ, 122, 3396

Grady, C. A., et al. 2009, ApJ, 699, 1822

Grady, C. A., et al. 2013, ApJ, 762, 48

Hashimoto, J., et al. 2011, ApJ, 729, L17

Honda, M., et al. 2015, ApJ, 804, 143

Hughes, A. M., et al. 2009, ApJ, 698, 131

Ingleby, L., Espaillat, C., Calvet, N., Sitko, M., Russell, R., \& Champney, E. 2015, ApJ, 805, 149

Isella, A., Natta, A., Wilner, D., Carpenter, J. M., \& Testi, L. 2010, ApJ, 725, 1735

Isella, A., Pérez, L. M., Carpenter, J. M., Ricci, L., Andrews, S., \& Rosenfeld, K. 2013, ApJ, 775, 30

Isella, A., Tatulli, E., Natta, A., \& Testi, L. 2008, A\&A, 483, L13

Jørgensen, J. K., Visser, R., Williams, J. P., \& Bergin, E. A. 2015, A\&A, 579, A23

Juhász, A., Benisty, M., Pohl, A., Dullemond, C. P., Dominik, C., \& Paardekooper, S.-J. 2015, MNRAS, 451, 1147

Kasper, M., Apai, D., Wagner, K., \& Robberto, M. 2015, ApJ, 812, L33

Lacour, S., et al. 2015, A\&A, submitted

Lin, S.-Y., Ohashi, N., Lim, J., Ho P. T. P., Fukagawa, M., \& Tamura, M. 2006, ApJ, 645, 1297

Looper, D. L., et al. 2010, ApJ, 714, 45

Lyra, W., \& Lin, M.-K. 2013, ApJ, 775, 17

Maaskant, K. M., et al. 2013, A\&A, 555, A64

Manset, N., Bastien, P., Ménard, F., Bertout, C., Le van Suu, A., \& Boivin, L. 2009, A\&A, 499, 137

Marino, S., Casassus, S., Perez, S., Lyra, W., Roman, P. E., Avenhaus, H., Wright, C. M., \& Maddison, S. T. 2015b, ApJ, 813, 76

Marino, S., Perez, S., \& Casassus, S. 2015a, ApJ, 798, L44

Martin, R. G., Nixon, C., Lubow, S. H., Armitage, P. J., Price, D. J., Doğan, S., \& King, A. 2014, ApJ, 792, L33

Matter, A., et al. 2016, A\&A, 586, A11

Mayama, S., et al. 2010, Sci, 327, 306

McGinnis, P. T., et al. 2015, A\&A, 577, A11

Menu, J., van Boekel, R., Henning, T., Leinert, C., Waelkens, C., \& Waters, L. B. F. M. 2015, A\&A, 581, A107

Mendigutía, I., de Wit, W. J, Oudmaijer, R. D, Fairlamb, J. R, Carciofi, A. C, Ilee, J. D, \& Vieira, R. G. 2015, MNRAS, 453, 2126

Millar-Blanchaer, M. A., et al. 2015, ApJ, 811, 18

Mittal, T., \& Chiang, E. 2015, ApJ, 798, L25

Montesinos, M., Cuadra, J., Perez, S., Baruteau, C., \& Casassus, S. 2015, ApJ, 806, 253

Montesinos, M., Perez, S., Casassus, S., Marino, S., Cuadra, J., \& Christiaens, V. 2016, arXiv:1601.07912

Mulders, G. D., Paardekooper, S.-J., Panić, O., Dominik, C., van Boekel, R., \& Ratzka, T. 2013, A\&A, 557, A68

Muto, T., et al. 2012, ApJ, 748, L22

Muto, T., et al. 2015, PASJ, 67, 122

Najita, J., Carr, J. S., \& Mathieu, R. D. 2003, ApJ, 589, 931

Nealon, R., Price, D. J., \& Nixon, C. J. 2015, MNRAS, 448, 1526

Nixon, C., King, A., \& Price, D. 2013, MNRAS, 434, 1946

Ober, F., Wolf, S., Uribe, A. L., \& Klahr, H. H. 2015, A\&A, 579, A105 
Ohashi, N. 2008, Ap\&SS, 313, 101

Olofsson, J. et al. 2013, A\&A, 552, A4

Owen, J. E. 2016, PASA, 33, 5

Paardekooper, S.-J., \& Mellema, G. 2006, A\&A, 453, 1129

Pérez, L. M., Isella, A., Carpenter, J. M., \& Chandler, C. J. 2014, ApJ, 783, L13

Perez, S., Dunhill, A., Casassus, S., Roman, P., Szulágyi, J., Flores, C., Marino, S., \& Montesinos, M. 2015b, ApJ, 811, L5

Perez, S., et al. 2015a, ApJ, 798, 85

Piétu, V., Guilloteau, S., \& Dutrey, A. 2005, A\&A, 443, 945

Pinilla, P., Benisty, M., \& Birnstiel, T. 2012, A\&A, 545, A81

Pinilla, P., et al. 2015, A\&A, 584, A16

Pinte, C., Dent, W. R. F., Ménard, F., Hales, A., Hill, T., Cortes, P., \& de Gregorio-Monsalvo, I. 2016, ApJ, 816, 25

Pohl, A., Pinilla, P., Benisty, M., Ataiee, S., Juhász, A., Dullemond, C. P., Van Boekel, R., \& Henning, T. 2015, MNRAS, 453, 1768

Pontoppidan, K. M., Blake, G. A., \& Smette, A. 2011, ApJ, 733, 84

Pontoppidan, K. M., Blake, G. A., van Dishoeck, E. F., Smette, A., Ireland, M. J., \& Brown, J. 2008, ApJ, 684, 1323

Quanz, S. P., Amara, A., Meyer, M. R., Girard, J. H., Kenworthy M. A., \& Kasper, M. 2015, ApJ, 807, 64

Quanz, S. P., Amara, A., Meyer, M. R., Kenworthy, M. A., Kasper, M., \& Girard, J. H. 2013, ApJ, 766, L1

Quillen, A. C. 2006, ApJ, 640, 1078

Rafikov, R. R. 2016, ApJ, submitted

Rameau, J., Chauvin, G., Lagrange, A.-M., Thébault, P., Milli, J., Girard, J. H., \& Bonnefoy, M. 2012, A\&A, 546, A24

Regály, Z., Juhász, A., Sándor, Z., \& Dullemond, C. P. 2012, MNRAS, 419, 1701

Reggiani, M., et al. 2014, ApJ, 792, L23

Roberge, A., Weinberger, A. J., \& Malumuth, E. M. 2005, ApJ, 622, 1171

Rodigas, T. J., Follette, K. B., Weinberger, A., Close, L., \& Hines D. C. 2014, ApJ, 791, L37

Rosenfeld, K. A., Chiang, E., \& Andrews, S. M. 2014, ApJ, 782, 62

Rosenfeld, K. A., et al. 2012, ApJ, 757, 129

Sallum, S., et al. 2015, Nature, 527, 342

Salyk, C., Blake, G. A., Boogert, A. C. A., \& Brown, J. M. 2009, ApJ, 699, 330

Salyk, C., Pontoppidan, K., Corder, S., Muñoz, D., Zhang, K., \& Blake, G. A. 2014, ApJ, 792, 68

Schisano, E., Covino, E., Alcalá, J. M., Esposito, M., Gandolfi D., \& Guenther, E. W. 2009, A\&A, 501, 1013
Schneider, P. C., France, K., Günther, H. M., Herczeg, G., Robrade, J., Bouvier, J., McJunkin, M., \& Schmitt, J. H. M. M. 2015, A\&A, 584, A51

Shu, F. H., Adams, F. C., \& Lizano, S. 1987, ARAA, 25, 23

Tang, Y.-W., Guilloteau, S., Piétu, V., Dutrey, A., Ohashi, N., \& Ho, P. T. P. 2012, A\&A, 547, A84

Tatulli, E., et al. 2011, A\&A, 531, A1

Testi, L., et al. 2014, Protostars and Planets VI, 339-361

Testi, L., et al. 2015, ApJ, 812, L38

Thi, W.-F., et al. 2014, A\&A, 561, A50

van Boekel, R., et al. 2004, Nature, 432, 479

van Dishoeck, E. F., van der Marel, N., Bruderer, S., \& Pinilla, P. 2016, MNRAS, accepted

van der Marel, N., et al. 2013, Science, 340, 1199

van der Marel, N., Pinilla, P., Tobin, J., van Kempen, T., Andrews S., Ricci, L., \& Birnstiel, T. 2015b, ApJ, 810, L7

van der Marel, N., van Dishoeck, E. F., Bruderer, S., Andrews, S. M., Pontoppidan, K. M., Herczeg, G. J., van Kempen, T., \& Miotello, A. 2016, A\&A, 585, A58

van der Marel, N., van Dishoeck, E. F., Bruderer, S., Pérez, L., \& Isella, A. 2015a, A\&A, 579, A106

van der Plas, G., Casassus, S., Ménard, F., Perez, S., Thi, W. F., Pinte, C., \& Christiaens, V. 2014, ApJ, 792, L25

van der Plas, G., van den Ancker, M. E., Acke, B., Carmona, A., Dominik, C., Fedele, D., \& Waters, L. B. F. M. 2009, A\&A, 500, 1137

van der Plas, G., van den Ancker, M. E., Fedele, D., Acke, B., Dominik, C., Waters, L. B. F. M., \& Bouwman, J. 2008, A\&A, 485,487

van der Plas, G., van den Ancker, M. E., Waters, L. B. F. M., \& Dominik C. 2015, A\&A, 574, A75

Wagner, K., Apai, D., Kasper, M., \& Robberto, M. 2015, ApJ, 813, L2

Wahhaj, Z., et al. 2015, A\&A, 581, A24

Wang, J. J., et al. 2015, ApJ, 811, L19

Weidenschilling, S. J. 1977, MNRAS, 180, 57

Whitney, B. A., Robitaille, T. P., Bjorkman, J. E., Dong, R., Wolff M. J., Wood, K., \& Honor, J. 2013, ApJS, 207, 30

Williams, J. P., \& Cieza, L. A. 2011, ARAA, 49, 67

Zhang, K., Isella, A., Carpenter, J. M., \& Blake, G. A. 2014, ApJ, 791, 42

Zhu, Z., \& Baruteau, C. 2015, preprint (arXiv 1511.03497)

Zhu, Z., \& Stone, J. M. 2014, ApJ, 795, 53

Zuckerman, B. 2001, ARAA, 39, 549 